\title{
Approximate analytical dynamical mean-field approach to strongly correlated electron systems
}

\author{
I.V.Stasyuk \\ Institute for Condensed Matter Physics \\ of the National Academy of Sciences of Ukraine, \\ 1 Svientsitskii Str., 79011 Lviv, Ukraine
}

Received March 17, 2000

\begin{abstract}
An approximate analytical scheme of the dynamical mean-field theory (DMFT), that is used for electron systems with Hubbard correlations and is exact in the limit of the infinite dimensionality of a space, is developed. The effective single-site problem arising in the framework of this method is formulated in terms of the auxiliary Fermi-field. The irreducible Green's function technique with the projecting on the Hubbard basis of Fermi-operators is used for its solution. A system of DMFT equations is obtained in the approximation which is a generalization of the Hubbard-III approximation and combines it with a self-consistent renormalization of the local electron levels. It is shown that the proposed approach includes as simple specific cases a number of known approximations (Hubbard-III, AA, MAA, ...) based on the assumption of the single-site structure of the electron selfenergy.
\end{abstract}

Key words: strong electron correlation, Hubbard model, dynamical mean-field theory, auxiliary Fermi-field, irreducible Green's functions

PACS: $71.10 . F d, 05.30 . F k$

This article is dedicated to Prof. Ihor Yukhnovskii on the occasion of his 75th birthday.

\section{Introduction}

Strongly correlated electron systems have been the subject of a growing attention of the investigators in recent years. Despite the relative simplicity of the models used for their description the theory of electron spectrum and thermodynamic properties of such systems is far from its final completion. The use of localized (atomic) basis 
of electron states is the general feature of the models. Corresponding Hamiltonians

$$
H=\sum_{i} H_{i}+\sum_{\langle i j\rangle} \sum_{\sigma} t_{i j} a_{i \sigma}^{+} a_{j \sigma}
$$

include, on the one hand the electron transfer (hopping) $t_{i j}$ between neighbouring sites (atoms) in the crystal lattice and on the other hand the short-range single-site electron correlations. It is primarily the on-site energy of Coulomb repulsion $U$ in the case of the Hubbard model and the models based on that one:

$$
H_{i}=U n_{i \uparrow} n_{i \downarrow}-\mu \sum_{\sigma} n_{i \sigma} .
$$

Models like (1), (2) can be solved exactly in two limiting cases: atomic limit $(t=0)$ and band electrons $(U=0)$. Near these extreme cases the expansions in terms of $t$ or $U$ are used, but the consistent formulation of the perturbation theory especially in the case of strong coupling is not a simple task. The case of an intermediate coupling $t \sim U$ is more complicated for consideration. In this region of parameter values, the splitting in the band electron spectrum and the metalinsulator transition takes place.

Due to the presence of strong electron correlations, a state of the electron system and its properties depend essentially on the mean electron concentration. At a different filling of electron states and depending on the relation between $t$ and $U$ parameters, the system can be paramagnetic or the transition into ferro- (antiferro-) magnetic phase can take place. In the case of a more complicated structure of the Hamiltonian $H_{i}$ (due to the allowance for the other, besides electron, degrees of freedom) or when the interaction is extended to the nearest neighbours in a lattice, the charge ordering can appear; the effects of phase separation become possible as well. The listed phenomena are the subject of study in the framework of various approaches and methods.

A new impulse in the investigations in this field is connected with the development of a new approach having its origin in works [1-3] where the study of the (1), (2)-type model in the limit of infinite dimensionality of space $(d=\infty)$ has been proposed. Due to principal simplifications taking place in this case in the perturbation series the possibility exists to obtain exact results using the scheme that corresponds to the well known coherent potential approximation (CPA) in the theory of disordered crystalline alloys. The rapidly developing corresponding method became known as the dynamical mean-field theory (DMFT).

The central point in this method is formulation and solution of the auxiliary single-site problem. An initial model is mapped on that one while considering one site characteristics of the electron spectrum, such as single-site electron Green's function (see [4-6], as well as the review [7]). In this case the separated lattice site is considered as placed in some effective environment. Since the processes of electron hopping from the atom and returning into the atom are taken into account, the mean field acting on the electron states of the atom possesses a dynamical nature. This field is described by the coherent potential $J_{\sigma}(\omega)$ that should be determined in a self-consistent way. 
Only in some simple cases the single-site problem can be solved analytically. In general, including the Hubbard model, the application of numerical or seminumerical (such as quantum Monte Carlo or exact diagonalization, see [7]) methods turns out to be necessary.

At the same time it is of interest to develop approximate analytic approaches to the solution of the single-site problem. Their application at that stage is more effective than at considering the full model (a short review of such attempts was given recently in $[8,9]$ ). The availability of the analytical (even of approximate) method is useful especially for new models as well as at the transition to the finite dimensionality of the system. The accuracy of approximation can be estimated relating to the results of numerical calculations.

In this work the approximate scheme of calculation of the electron Green's function for the auxiliary single-site problem is proposed. The approach is based on the technique of the irreducible Green's functions. The procedure of projecting onto the basic set of operators is used (the set consists of the single-site electron Hubbard operators of the Fermi-type).

The recipe is given for the construction of the system of equations for the coherent potential and self-consistency parameter (having the meaning of a static part of the effective internal field) in the approach that is a generalization of the HubbardIII approximation. Specific cases are considered corresponding to the more simple approximations of the alloy-analogy (AA) or modified alloy-analogy (MAA) type $[10,11]$ in the DMFT method as well as to the certain decoupling procedure in the two-time Green's function method when applied to the initial electron problem.

\section{Hubbard model and similar models in a limit of infinite di- mension of space $(d=\infty)$}

The transition to the $d=\infty$ in the DMFT approach is accompanied by the scaling of the electron transfer parameter

$$
t=\frac{t^{*}}{\sqrt{d}}
$$

In the case of $d$-dimensional hypercubic lattice with an electron spectrum

$$
\varepsilon_{k}=\frac{2 t^{*}}{\sqrt{d}} \sum_{\alpha=1}^{d} \cos k_{\alpha} a
$$

this procedure leads to the Gaussian density of electron states [2]

$$
\rho_{0}(\omega)=\frac{1}{2 \sqrt{\pi} t^{*}} \mathrm{e}^{-\frac{\omega^{2}}{4 t^{* 2}}}
$$

The average kinetic energy remains constant in this case in the limit $d=\infty$.

The scaling (3) has a significant effect on the structure of diagrammatic series for single-electron Green's functions of the model of the (1) and (2) type. In particular, 
the irreducible self-energy part of such a function becomes a purely local (a singlesite) quantity $[2,3]$ :

$$
\Sigma_{i j, \sigma}(\omega)=\Sigma_{\sigma}(\omega) \delta_{i j}, \quad d=\infty .
$$

The Fourier-transform of $\Sigma_{i j, \sigma}(\omega)$ is hence momentum-independent

$$
\Sigma_{\sigma}(\vec{k}, \omega)=\Sigma_{\sigma}(\omega) .
$$

This leads to tremendous simplifications in all many-body calculations for the Hubbard model and related models and enables us to obtain the exact numerical results for the main parameters of the electron spectrum, to describe magnetic phase transitions and the metal-insulator transformation etc. (see, for example, $[7,9]$ ).

The possibility of obtaining exact solutions in the $d=\infty$ limit opens the way to the development of a theory based on the expansion in powers of $1 / d$ (the results for $d=\infty$ can be considered as zero approximation in this case). Such approaches have been elaborated for the last few years $[12,13]$. On the other hand, consideration in the framework in the $d=\infty$ limit is not only of an academic interest. It turns out that a set of the known approximating schemes or methods is correct in the $d=\infty$ limit. Besides, the obtained physical conclusions can be transferred in many cases to the system with finite dimensions keeping their suitability even at $d=3$.

The formal scheme of calculating the electron Green's functions and the main thermodynamical quantities can be developed basing on the diagrammatic expansions in powers of interaction parameters (such as energy $U$ in the case of the Hubbard model) or matrix elements of the electron transfer $t_{i j}$. The electron Green's function in $(\vec{k}, \omega)$ representation

$$
G_{k}^{\sigma}(\omega)=\sum_{i-j} \mathrm{e}^{\mathrm{i} \vec{k}\left(\vec{R}_{i}-\vec{R}_{j}\right)} G_{i j, \sigma}(\omega)
$$

can be expressed in the first or in the second of these cases as

$$
G_{k}^{\sigma}(\omega)=\frac{1}{\omega+\mu-t_{k}-\Sigma_{\sigma}(\omega)}
$$

or

$$
G_{k}^{\sigma}(\omega)=\frac{1}{\left[\Xi_{\sigma}(\omega)\right]^{-1}-t_{k}},
$$

where $\Sigma_{\sigma}(\omega)$ or $\Xi_{\sigma}(\omega)$ are the self-energy parts, which are irreducible (in the diagrammatic representation) according to Dyson or Larkin, respectively.

To calculate the $\Sigma_{\sigma}(\omega)$ (or $\Xi_{\sigma}(\omega)$ ) function, the effective single-site problem is used. As was shown in [14], the transition to this problem corresponds to the replacement

$$
\mathrm{e}^{-\beta H} \rightarrow \mathrm{e}^{-\beta H_{\mathrm{eff}}}=\mathrm{e}^{-\beta H_{0}} T \exp \left\{-\int_{0}^{\beta} \mathrm{d} \tau \int_{0}^{\beta} \mathrm{d} \tau^{\prime} \sum_{\sigma} J_{\sigma}\left(\tau-\tau^{\prime}\right) a_{\sigma}^{+}(\tau) a_{\sigma}\left(\tau^{\prime}\right)\right\},
$$

where

$$
H_{0}=H_{i}
$$


and $J_{\sigma}\left(\tau-\tau^{\prime}\right)$ is an effective auxiliary field which is determined self-consistently from the condition that the same self-energy part $\Xi_{\sigma}(\omega)$ determines the lattice function (10) as well as the Green's function $G_{\sigma}^{(a)}(\omega)$ of the effective single-site problem. The last one is connected with $\Xi_{\sigma}(\omega)$ and $J_{\sigma}(\omega)$ by the relation

$$
G_{\sigma}^{(a)}(\omega)=\frac{1}{\left[\Xi_{\sigma}(\omega)\right]^{-1}-J_{\sigma}(\omega)}
$$

In this case

$$
G_{\sigma}^{(a)}(\omega)=G_{i i, \sigma}(\omega)=\frac{1}{N} \sum_{k} G_{k}^{\sigma}(\omega)
$$

Dynamical field $J_{\sigma}\left(\tau-\tau^{\prime}\right)$ describes electron hopping from the given site into the surroundings and vice versa; the electron propagates in the environment without going through this site between moments $\tau$ and $\tau^{\prime}$. The expression

$$
G_{\sigma}(\omega)=\sum_{k j} t_{i k} t_{i j} G_{k j, \sigma}^{(i)}
$$

corresponds to this situation (the relation (15) is known from the standard CPA scheme $[15,16])$; here $G_{k j, \sigma}^{(i)}$ is the electron Green's function for a crystal with the excluded site $i$.

The set of simultaneous equations (10), (13) and (14) becomes closed when it is supplemented by the functional dependence

$$
G_{\sigma}^{(a)}(\omega)=f\left(\left[J_{\sigma}(\omega)\right]\right)
$$

which is obtained as the result of solving the effective single-site problem with the statistical operator $\exp \left(-\beta H_{\text {eff }}\right)$. It is possible to do this in an analytical way only in some cases of simple models (a Falicov-Kimball model [17]; a pseudospin-electron model at $U=0$ [18]; a usual binary alloy model). In general, numerical methods are used.

The scheme described lies at the basis of the above mentioned DMFT approach used in the last years in describing strongly correlated electron systems.

\section{Electron Green's functions of the effective single-site problem}

As it was mentioned, the central point in the DMFT approach is the solution of the effective single-site problem and the determination of the connection between the dynamical mean field (coherent potential) $J_{\sigma}(\omega)$ and the single-site electron Green's function $G_{\sigma}^{(a)}(\omega)$. We propose in this work an approximate scheme, which is based on the technique of the irreducible two-time temperature Green's functions and leads to the results having an interpolating character. The Hubbard model is taken into consideration to illustrate the method. 
Let us reformulate a single-site problem introducing explicitly an effective Hamiltonian

$$
\tilde{H}_{\mathrm{eff}}=H_{0}+V \sum_{\sigma}\left(a_{\sigma}^{+} \xi_{\sigma}+\xi_{\sigma}^{+} a_{\sigma}\right)+H_{\xi},
$$

where the auxiliary Fermi-field is brought in. It describes the environment of the selected site and formally is characterized by the Hamiltonian $H_{\xi}$. The single-electron transitions between the site and the environment are taken into account.

An explicit form of the Hamiltonian $H_{\xi}$ is unknown. Let us consider, however, the Green's function

$$
\mathcal{G}_{\sigma}(\omega)=\left\langle\left\langle\xi_{\sigma} \mid \xi_{\sigma}^{+}\right\rangle\right\rangle_{\omega}^{\left(H_{\xi}\right)}
$$

for auxiliary fermions as the given function. The function $\mathcal{G}_{\sigma}\left(\tau-\tau^{\prime}\right)=\left\langle T_{\tau} \xi_{\sigma}^{+}(\tau) \xi_{\sigma}\left(\tau^{\prime}\right)\right\rangle_{0}$ (where averaging is performed with the part $H_{\xi}$ of the Hamiltonian (17)) corresponds to the function (18) in the Matsubara's representation. It is shown in appendix A that the expansion of the $\exp \left(-\beta \tilde{H}_{\text {eff }}\right)$ operator in powers of $V$ and the subsequent averaging over the states of $\xi$-subsystem using the Wick's theorem and the functions (18) leads to the statistical operator (11):

$$
\left\langle\exp \left(-\beta \tilde{H}_{\mathrm{eff}}\right)\right\rangle^{\left(H_{\xi}\right)}=\exp \left(-\beta H_{\mathrm{eff}}\right) .
$$

The relation

$$
2 \pi V^{2} \mathcal{G}_{\sigma}(\omega)=J_{\sigma}(\omega)
$$

takes place in this case.

The obtained result points out to the possibility of the Green's function $G_{\sigma}^{(a)}(\omega)$ calculation based on the Hamiltonian $\tilde{H}_{\text {eff }}$. The averaging over the $a, a^{+}$-variables is performed with the use of the Gibbs distribution while over the $\xi, \xi^{+}$-variables it is done with the help of function (18).

Let us write the Hamiltonian (17) for the case of the Hubbard model in terms of Hubbard operators

$$
\begin{aligned}
\tilde{H}_{\mathrm{eff}}= & -\mu\left(\sum_{\sigma} X^{\sigma \sigma}+2 X^{22}\right)+U X^{22} \\
& +V \sum_{\sigma}\left[\left(X^{\sigma 0}+\sigma X^{2,-\sigma}\right) \xi_{\sigma}+\xi_{\sigma}^{+}\left(X^{0 \sigma}+\sigma X^{-\sigma, 2}\right)\right]+H_{\xi} .
\end{aligned}
$$

Here the standard basis of single-site states is used $(\sigma=+,-)$

$$
\begin{array}{ll}
|0\rangle=\left|\left(1-n_{\uparrow}\right)\left(1-n_{\downarrow}\right)\right\rangle, & |-\rangle=\left|\left(1-n_{\uparrow}\right) n_{\downarrow}\right\rangle, \\
|2\rangle=\left|n_{\uparrow} n_{\downarrow}\right\rangle, & |+\rangle=\left|n_{\uparrow}\left(1-n_{\downarrow}\right)\right\rangle .
\end{array}
$$

In this case the Green's function $G_{\sigma}^{(a)}(\omega)$ can be written in the form

$$
G_{\sigma}^{(a)}=\left\langle\left\langle X^{0 \sigma} \mid X^{\sigma 0}\right\rangle\right\rangle_{\omega}+\sigma\left\langle\left\langle X^{0 \sigma} \mid X^{2,-\sigma}\right\rangle\right\rangle_{\omega}+\sigma\left\langle\left\langle X^{-\sigma, 2} \mid X^{\sigma 0}\right\rangle\right\rangle_{\omega}+\left\langle\left\langle X^{-\sigma, 2} \mid X^{2,-\sigma}\right\rangle\right\rangle_{\omega}
$$

(a representation in terms of two-time Green's functions is used). 
We will write the equations for functions (23) using the equations of motion for $X$-operators:

$$
\begin{aligned}
\mathrm{i} \frac{\mathrm{d}}{\mathrm{d} t} X^{0 \sigma}(t)=\left[X^{0 \sigma}, \tilde{H}_{\text {eff }}\right]= & -\mu X^{0 \sigma}+V\left(X^{00}+X^{\sigma \sigma}\right) \xi_{\sigma} \\
& +V X^{-\sigma, \sigma} \xi_{-\sigma}+\sigma V X^{02} \xi_{-\sigma}^{+}, \\
\mathrm{i} \frac{\mathrm{d}}{\mathrm{d} t} X^{-\sigma, 2}(t)=\left[X^{-\sigma, 2} \tilde{H}_{\text {eff }}\right]= & (U-\mu) X^{-\sigma, 2}+\sigma V\left(X^{22}+X^{-\sigma,-\sigma}\right) \xi_{\sigma} \\
& -\sigma V X^{-\sigma, \sigma} \xi_{-\sigma}-V X^{02} \xi_{-\sigma}^{+} .
\end{aligned}
$$

As a result, we have

$$
\begin{aligned}
(\omega+\mu)\left\langle\left\langle X^{0 \sigma} \mid X^{\sigma 0}\right\rangle\right\rangle= & \frac{1}{2 \pi} A_{0 \sigma}+V\left\langle\left\langle\left(X^{00}+X^{\sigma \sigma}\right) \xi_{\sigma} \mid X^{\sigma 0}\right\rangle\right\rangle \\
& +V\left\langle\left\langle X^{-\sigma, \sigma} \xi_{-\sigma} \mid X^{\sigma 0}\right\rangle\right\rangle+\sigma V\left\langle\left\langle X^{02} \xi_{-\sigma}^{+} \mid X^{\sigma 0}\right\rangle\right\rangle, \\
(\omega+\mu-U)\left\langle\left\langle X^{-\sigma, 2} \mid X^{\sigma 0}\right\rangle\right\rangle= & \sigma V\left\langle\left\langle\left(X^{22}+X^{-\sigma,-\sigma}\right) \xi_{\sigma} \mid X^{\sigma 0}\right\rangle\right\rangle \\
& -\sigma V\left\langle\left\langle X^{-\sigma, \sigma} \xi_{-\sigma} \mid X^{\sigma 0}\right\rangle\right\rangle-V\left\langle\left\langle X^{02} \xi_{-\sigma}^{+} \mid X^{\sigma 0}\right\rangle\right\rangle,
\end{aligned}
$$

where $A_{p q}=\left\langle X^{p p}+X^{q q}\right\rangle ; A_{0 \sigma}=1-n_{-\sigma}, A_{-\sigma, 2}=n_{-\sigma}$.

Let us separate in Green's functions of higher order the irreducible parts using the method developed in $[19,20]$. Proceeding from the equations of motion (24) we express derivatives $\mathrm{i} \frac{\mathrm{d}}{\mathrm{d} t} X^{0 \sigma(-\sigma, 2)}$ as a sum of regular (projected on the subspace formed by operators $X^{0 \sigma}, X^{-\sigma, 2}$ ) and irregular parts. The latter ones describe an inelastic quasiparticle scattering. We obtain

$$
\begin{aligned}
{\left[X^{0 \sigma}, \tilde{H}_{\mathrm{eff}}\right] } & =-\mu X^{0 \sigma}+\alpha_{1}^{0 \sigma} X^{0 \sigma}+\alpha_{2}^{0 \sigma} X^{-\sigma, 2}+Z^{0 \sigma} \\
{\left[X^{-\sigma, 2}, \tilde{H}_{\mathrm{eff}}\right] } & =(U-\mu) X^{-\sigma, 2}+\alpha_{1}^{-\sigma, 2} X^{0 \sigma}+\alpha_{2}^{-\sigma, 2} X^{-\sigma, 2}+Z^{-\sigma, 2} .
\end{aligned}
$$

Operators $Z^{0 \sigma}$ and $Z^{-\sigma, 2}$ are defined as orthogonal ones to operators from the basic subspace:

$$
\begin{aligned}
\left\langle\left\{Z^{0 \sigma(-\sigma, 2)}, X^{\sigma 0}\right\}\right\rangle & =0, \\
\left\langle\left\{Z^{0 \sigma(-\sigma, 2)}, X^{2,-\sigma}\right\}\right\rangle & =0 .
\end{aligned}
$$

These equations determine the coefficients $\alpha_{i}^{0 \sigma(-\sigma, 2)}$.

Using the described procedure we come to the expressions

$$
\begin{aligned}
& Z^{0 \sigma}=V \overparen{\left(X^{00}+X^{\sigma \sigma}\right) \xi_{\sigma}}+V \overparen{X^{-\sigma, \sigma} \xi_{-\sigma}}+\sigma V \overparen{X^{02} \xi_{-\sigma}^{+}}, \\
& Z^{-\sigma, 2}=\sigma V \overparen{\left(^{22}+X^{-\sigma,-\sigma}\right) \xi_{\sigma}}-\sigma V \overparen{X^{-\sigma, \sigma} \xi_{-\sigma}}-V \overparen{X^{02} \xi_{-\sigma}^{+}},
\end{aligned}
$$

where

$$
\begin{aligned}
& \overparen{\left(X^{00}+X^{\sigma \sigma}\right) \xi_{\sigma}}=\left(X^{00}+X^{\sigma \sigma}\right) \xi_{\sigma}, \\
& \overparen{\left(X^{22}+X^{-\sigma,-\sigma}\right) \xi_{\sigma}}=\left(X^{22}+X^{-\sigma,-\sigma}\right) \xi_{\sigma}, \\
& \overparen{X^{-\sigma, \sigma} \xi_{-\sigma}}=X^{-\sigma, \sigma} \xi_{-\sigma}-\frac{1}{A_{0 \sigma}}\left\langle\xi_{-\sigma} X^{-\sigma, 0}\right\rangle X^{0 \sigma}-\frac{1}{A_{2,-\sigma}}\left\langle X^{2 \sigma} \xi_{-\sigma}\right\rangle X^{-\sigma, 2}, \\
& \overparen{X^{02} \xi_{-\sigma}^{+}}=X^{02} \xi_{-\sigma}^{+}-\frac{1}{A_{0 \sigma}}\left\langle X^{\sigma 2} \xi_{-\sigma}^{+}\right\rangle X^{0 \sigma}-\frac{1}{A_{2,-\sigma}}\left\langle\xi_{-\sigma}^{+} X^{0,-\sigma}\right\rangle X^{-\sigma, 2} .
\end{aligned}
$$


In this case

$$
\begin{aligned}
\alpha_{1}^{0 \sigma} & =-\sigma \alpha_{1}^{-\sigma, 2}=\frac{V}{A_{0 \sigma}} \varphi_{\sigma}, \\
\alpha_{2}^{0 \sigma} & =-\sigma \alpha_{2}^{-\sigma, 2}=-\frac{V}{A_{2,-\sigma}} \sigma \varphi_{\sigma}, \\
\varphi_{\sigma} & =\left\langle\xi_{-\sigma} X^{-\sigma, 0}\right\rangle+\sigma\left\langle X^{\sigma 2} \xi_{-\sigma}^{+}\right\rangle .
\end{aligned}
$$

(here we put $\varphi_{\sigma}=\varphi_{\sigma}^{*}$ ).

The set of equations (25) can be rewritten now in the form

$$
\left(\begin{array}{cc}
\omega-a_{\sigma} & \sigma \frac{V}{A_{2,-\sigma}} \varphi_{\sigma} \\
\sigma \frac{V}{A_{0 \sigma}} \varphi_{\sigma} & \omega-b_{\sigma}
\end{array}\right)\left(\begin{array}{c}
\left\langle\left\langle X^{0 \sigma} \mid X^{\sigma 0}\right\rangle\right\rangle \\
\left\langle\left\langle X^{-\sigma, 2} \mid X^{\sigma 0}\right\rangle\right\rangle
\end{array}\right)=\left(\begin{array}{c}
\frac{A_{0 \sigma}}{2 \pi}+\left\langle\left\langle Z^{0 \sigma} \mid X^{\sigma 0}\right\rangle\right\rangle \\
\left\langle\left\langle Z^{-\sigma, 2} \mid X^{\sigma 0}\right\rangle\right\rangle
\end{array}\right)
$$

where a notation is used:

$$
\begin{aligned}
& a_{\sigma}=-\mu+\frac{V}{A_{0 \sigma}} \varphi_{\sigma}, \\
& b_{\sigma}=-\mu+U+\frac{V}{A_{2,-\sigma}} \varphi_{\sigma} .
\end{aligned}
$$

An equation for Green's function $\left\langle\left\langle Z^{0 \sigma(-\sigma, 2)} \mid X^{\sigma 0}\right\rangle\right\rangle$ can be obtained by means of differentiation with respect to the second time argument:

$$
\begin{aligned}
& \left\langle\left\langle Z^{0 \sigma(-\sigma, 2)} \mid X^{\sigma 0}\right\rangle\right\rangle(\omega+\mu)= \\
& \quad=\left\langle\left\langle Z^{0 \sigma(-\sigma, 2)} \mid X^{\sigma, 0}\right\rangle\right\rangle \alpha_{1}^{0 \sigma}+\left\langle\left\langle Z^{0 \sigma(-\sigma, 2)} \mid X^{2,-\sigma}\right\rangle\right\rangle \alpha_{2}^{0 \sigma}+\left\langle\left\langle Z^{0 \sigma(-\sigma, 2)} \mid Z^{\sigma 0}\right\rangle\right\rangle .
\end{aligned}
$$

The similar procedure can be applied to the functions containing the operator $X^{2,-\sigma}$ on the right side. Joining them together with the previous ones into a single matrix Green's function

$$
\hat{G}=2 \pi\left(\begin{array}{cc}
\left\langle\left\langle X^{0 \sigma} \mid X^{\sigma 0}\right\rangle\right\rangle & \left\langle\left\langle X^{0 \sigma} \mid X^{2,-\sigma}\right\rangle\right\rangle \\
\left\langle\left\langle X^{-\sigma, 2} \mid X^{\sigma 0}\right\rangle\right\rangle & \left\langle\left\langle X^{-\sigma 2} \mid X^{2,-\sigma}\right\rangle\right\rangle
\end{array}\right)
$$

we can write the following equation, which corresponds to (31) and (33):

$$
\hat{G}=\hat{G}_{0}+\hat{G}_{0} \hat{P}_{\sigma} \hat{G}_{0}
$$

Here the nonperturbed Green's function $\hat{G}_{0}$ is introduced

$$
\hat{G}_{0}=\frac{1}{D_{\sigma}}\left(\begin{array}{cc}
\omega-b_{\sigma} & -\sigma \frac{V}{A_{2,-}} \varphi_{\sigma} \\
-\sigma \frac{V}{A_{0 \sigma}} \varphi_{\sigma} & \omega-a_{\sigma}
\end{array}\right)
$$

where

$$
D_{\sigma}=\left(\omega-a_{\sigma}\right)\left(\omega-b_{\sigma}\right)-\frac{V^{2}}{A_{0 \sigma} A_{2,-\sigma}} \varphi_{\sigma}^{2}
$$


the matrix

$$
\hat{P}_{\sigma}=2 \pi\left(\begin{array}{cc}
A_{0 \sigma}^{-1} & 0 \\
0 & A_{2,-\sigma}^{-1}
\end{array}\right)\left(\begin{array}{cc}
\left\langle\left\langle Z^{0 \sigma} \mid Z^{\sigma 0}\right\rangle\right\rangle & \left\langle\left\langle Z^{0 \sigma} \mid Z^{2,-\sigma}\right\rangle\right\rangle \\
\left\langle\left\langle Z^{-\sigma, 2} \mid Z^{\sigma 0}\right\rangle\right\rangle & \left\langle\left\langle Z^{-\sigma, 2} \mid Z^{2,-\sigma}\right\rangle\right\rangle
\end{array}\right)\left(\begin{array}{cc}
A_{0 \sigma}^{-1} & 0 \\
0 & A_{2,-\sigma}^{-1}
\end{array}\right)
$$

has the meaning of a scattering matrix. Being expressed in terms of irreducible Green's functions it contains the scattering corrections of the second and the higher order in powers of $V$. The separation in $\hat{P}$ of the irreducible, with respect to $V$, parts enables us to obtain a mass operator $\hat{M}$

$$
\begin{aligned}
\hat{P}_{\sigma} & =\hat{M}_{\sigma}+\hat{M}_{\sigma} \hat{G}_{0} \hat{M}_{\sigma}+\hat{M}_{\sigma} \hat{G}_{0} \hat{M}_{\sigma} \hat{G}_{0} \hat{M}_{\sigma}+\ldots, \\
\hat{M}_{\sigma} & =\left.\hat{P}_{\sigma}\right|_{\text {ir }} .
\end{aligned}
$$

In this case the relation (35) can be transformed into Dyson equation

$$
\hat{G}=\hat{G}_{0}+\hat{G}_{0} \hat{M}_{\sigma} \hat{G}
$$

with the solution

$$
\hat{G}=\left(1-\hat{G}_{0} \hat{M}_{\sigma}\right)^{-1} \hat{G}_{0},
$$

which provides a formed final expression for the Green's function (34).

\section{Different-time decoupling of irreducible Green's functions}

We will restrict ourselves hereafter to the simple approximation in calculating the mass operator $\hat{P}$, taking into account the scattering processes of the second order in $V$. In this case

$$
\hat{M}_{\sigma}=\hat{P}_{\sigma}^{(0)},
$$

where the irreducible Green's functions are calculated without allowance for correlation between electron transitions on the given site and environment. It corresponds to the procedure of the different-time decoupling [21], which means in our case an independent averaging of products of $X$ and $\xi$ operators.

Let us illustrate this approximation with some examples.

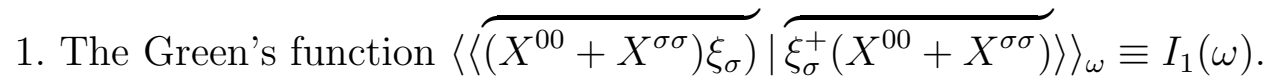
According to the spectral theorem we have

$I_{1}(\omega)=\frac{1}{2 \pi} \int_{-\infty}^{+\infty} \frac{\mathrm{d} \omega^{\prime}}{\omega-\omega^{\prime}}\left(\mathrm{e}^{\beta \omega^{\prime}}+1\right) \int_{-\infty}^{+\infty} \frac{\mathrm{d} t}{2 \pi} \mathrm{e}^{-i \omega^{\prime} t}\left\langle\xi_{\sigma}^{+}(t)\left(X^{00}+X^{\sigma \sigma}\right)_{t}\left(X^{00}+X^{\sigma \sigma}\right) \xi_{\sigma}\right\rangle^{\mathrm{ir}}$.

Due to different-time decoupling

$$
\left\langle\xi_{\sigma}^{+}(t)\left(X^{00}+X^{\sigma \sigma}\right)_{t}\left(X^{00}+X^{\sigma \sigma}\right) \xi_{\sigma}\right\rangle^{\mathrm{ir}} \approx\left\langle\left(X^{00}+X^{\sigma \sigma}\right)_{t}\left(X^{00}+X^{\sigma \sigma}\right)\right\rangle\left\langle\xi_{\sigma}^{+}(t) \xi_{\sigma}\right\rangle .
$$

We will take the first of these correlators in a zero approximation

$$
\left\langle\left(X^{00}+X^{\sigma \sigma}\right)_{t}\left(X^{00}+X^{\sigma \sigma}\right)\right\rangle \approx\left\langle\left(X^{00}+X^{\sigma \sigma}\right)^{2}\right\rangle=A_{0 \sigma}
$$


and substitution of (44) into (43) leads in this case to the result

$$
I_{1}(\omega)=A_{0 \sigma}\left\langle\left\langle\xi_{\sigma} \mid \xi_{\sigma}^{+}\right\rangle\right\rangle_{\omega} .
$$

2. The Green's function $\left\langle\langle\overbrace{X^{-\sigma, \sigma} \xi_{-\sigma}} \mid \overbrace{\xi_{-\sigma}^{+} X^{\sigma,-\sigma}}\rangle\right\rangle_{\omega} \equiv I_{2}(\omega)$.

The representation of the $I_{2}(\omega)$ function in the form analogous to (43) leads to the time correlation function $\left\langle\xi_{-\sigma}^{+}(t) X^{\sigma,-\sigma}(t) X^{-\sigma \sigma} \xi_{-\sigma}\right\rangle^{\text {ir }}$ that can be approximated as

$$
\left\langle\xi_{-\sigma}^{+}(t) X^{\sigma,-\sigma}(t) X^{-\sigma, \sigma} \xi_{-\sigma}\right\rangle^{\mathrm{ir}} \approx\left\langle X^{\sigma,-\sigma}(t) X^{-\sigma, \sigma}\right\rangle\left\langle\xi_{-\sigma}^{+}(t) \xi_{-\sigma}\right\rangle
$$

and, respectively

$$
\left\langle X^{\sigma,-\sigma}(t) X^{-\sigma \sigma}\right\rangle \approx\left\langle X^{\sigma \sigma}\right\rangle
$$

In this case

$$
I_{2}(\omega)=\left\langle X^{\sigma \sigma}\right\rangle\left\langle\left\langle\xi_{-\sigma} \mid \xi_{-\sigma}^{+}\right\rangle\right\rangle_{\omega}
$$

3. The Green's function $\left\langle\langle\overbrace{X^{02} \xi_{-\sigma}^{+}} \mid \overbrace{\xi_{-\sigma} X^{20}}\rangle\right\rangle_{\omega} \equiv I_{3}(\omega)$.

The corresponding time correlation function is decoupled as

$$
\left\langle\xi_{-\sigma}(t) X^{20}(t) X^{02} \xi_{-\sigma}^{+}\right\rangle^{\mathrm{ir}} \approx\left\langle X^{20}(t) X^{02}\right\rangle\left\langle\xi_{-\sigma}(t) \xi_{-\sigma}^{+}\right\rangle .
$$

In the zero approximation

$$
\left\langle X^{20}(t) X^{02}\right\rangle=\exp [\mathrm{i}(U-2 \mu) t]\left\langle X^{22}\right\rangle .
$$

Using these expressions we obtain

$$
\begin{aligned}
I_{3}(\omega) & =\frac{1}{2}\left\langle X^{00}+X^{22}\right\rangle\left\langle\left\langle\xi_{-\sigma}^{+} \mid \xi_{-\sigma}\right\rangle\right\rangle_{\omega+2 \mu-U} \\
+ & \left\langle X^{00}-X^{22}\right\rangle \frac{1}{2 \pi} \int_{-\infty}^{+\infty} \frac{\mathrm{d} \omega^{\prime}}{\omega+2 \mu-U-\omega^{\prime}} \operatorname{th} \frac{\beta \omega^{\prime}}{2}\left[-2 \operatorname{Im}\left\langle\left\langle\xi_{-\sigma}^{+} \mid \xi_{-\sigma}\right\rangle\right\rangle_{\omega^{\prime}+\mathrm{i} \varepsilon}\right] .
\end{aligned}
$$

Let us mention that at the half-filling of electron states (when $n=1,\left\langle X^{00}\right\rangle=$ $\left.\left\langle X^{22}\right\rangle\right)$

$$
I_{3}(\omega)=-\left\langle X^{22}\right\rangle\left\langle\left\langle\xi_{-\sigma} \mid \xi_{-\sigma}^{+}\right\rangle\right\rangle_{U-2 \mu-\omega} .
$$

Following the described procedure and taking into account the relation (20) we will come to the following expressions for irreducible Green's functions:

$$
\begin{aligned}
\left\langle\left\langle Z^{0 \sigma} \mid Z^{\sigma 0}\right\rangle\right\rangle_{\omega} & =A_{0 \sigma} J_{\sigma}(\omega)-R_{\sigma}(\omega), \\
\left\langle\left\langle Z^{-\sigma, 2} \mid Z^{2,-\sigma}\right\rangle\right\rangle_{\omega} & =A_{2,-\sigma} J_{\sigma}(\omega)-R_{\sigma}(\omega), \\
\left\langle\left\langle Z^{0 \sigma} \mid Z^{2,-\sigma}\right\rangle\right\rangle_{\omega} & =\left\langle\left\langle Z^{-\sigma, 2} \mid Z^{\sigma 0}\right\rangle\right\rangle_{\omega}=R_{\sigma}(\omega),
\end{aligned}
$$

where

$$
\begin{aligned}
& R_{\sigma}(\omega)=-\left\langle X^{\sigma \sigma}\right\rangle J_{-\sigma}(\omega)+\frac{1}{2}\left\langle X^{00}+X^{22}\right\rangle J_{-\sigma}(U-2 \mu-\omega) \\
& +\left\langle X^{00}-X^{22}\right\rangle \frac{1}{2 \pi} \int_{-\infty}^{+\infty} \frac{\operatorname{th} \beta \omega^{\prime} / 2}{\omega+2 \mu-U-\omega^{\prime}}\left[-2 \operatorname{Im} J_{-\sigma}\left(-\omega^{\prime}-\mathrm{i} \varepsilon\right)\right] \mathrm{d} \omega^{\prime} .
\end{aligned}
$$




\section{Basic set of equations}

Using the results obtained in the previous section we can write the expressions for mass operator components

$$
\begin{gathered}
M_{\sigma, 11}=\frac{1}{A_{0 \sigma}} J_{\sigma}(\omega)-\frac{1}{A_{0 \sigma}^{2}} R_{\sigma}(\omega), \quad M_{\sigma, 22}=\frac{1}{A_{2,-\sigma}} J_{\sigma}(\omega)-\frac{1}{A_{2,-\sigma}^{2}} R_{\sigma}(\omega), \\
M_{\sigma, 12}=M_{\sigma, 21}=\frac{1}{A_{0 \sigma} A_{2,-\sigma}} R_{\sigma}(\omega) .
\end{gathered}
$$

It follows from the relation (41) that

$$
\sum_{\alpha \beta} G_{\sigma, \alpha \beta}(\omega) \equiv G_{\sigma}^{(a)}(\omega)=\frac{\sum_{\alpha \beta} G_{\alpha \beta}^{0}+\operatorname{det}\left\|G^{0}\right\|\left(M_{\sigma, 12}+M_{\sigma, 21}-M_{\sigma, 11}-M_{\sigma, 22}\right)}{1-\sum_{\alpha \beta} G_{\alpha \beta}^{0} M_{\sigma, \beta \alpha}+\operatorname{det}\left\|G^{0}\right\| \operatorname{det}\left\|M_{\sigma}\right\|} .
$$

Substitution of expressions (36) and (56) into (57) leads to the result

$$
\begin{aligned}
\left(\sum_{\alpha \beta} G_{\alpha \beta}^{\sigma}\right)^{-1}= & {\left[\left(\omega-a_{\sigma}\right)\left(\omega-b_{\sigma}\right)-\left(\omega-a_{\sigma}\right)\left(J_{\sigma}-\frac{R_{\sigma}}{A_{2,-\sigma}}\right)-\left(\omega-b_{\sigma}\right)\left(J_{\sigma}-\frac{R_{\sigma}}{A_{0 \sigma}}\right)\right.} \\
& \left.+J_{\sigma}^{2}-\left(2 V \varphi_{\sigma} R_{\sigma}-V^{2} \varphi_{\sigma}^{2}-J_{\sigma} R_{\sigma}\right) \frac{1}{A_{0 \sigma} A_{2,-\sigma}}\right] \\
& \left.\times\left[\left(\omega-a_{\sigma}\right) A_{2,-\sigma}+\left(\omega-b_{\sigma}\right) A_{0 \sigma}-2 V \varphi_{\sigma}-J_{\sigma}+\frac{R_{\sigma}}{A_{0 \sigma} A_{2,-\sigma}}\right]^{-1} \cdot .58\right)
\end{aligned}
$$

Let us now use the relation

$$
\left[\Xi_{\sigma}(\omega)\right]^{-1}=\left(\sum_{\alpha \beta} G_{\alpha \beta}^{\sigma}\right)^{-1}+J_{\sigma}
$$

which follows from (13), to determine the single-site self-energy part. We obtain

$$
\Xi_{\sigma}(\omega)=\frac{\omega-\varepsilon_{+} A_{0 \sigma}-\varepsilon_{-} A_{2,-\sigma}-\tilde{\Omega}_{\sigma}(\omega)}{\left(\omega-\varepsilon_{+}\right)\left(\omega-\varepsilon_{-}\right)-\omega \tilde{\Omega}_{\sigma}(\omega)+\left(\varepsilon_{+} A_{2,-\sigma}+\varepsilon_{-} A_{0 \sigma}\right) \tilde{\Omega}_{\sigma}(\omega)},
$$

where $\varepsilon_{+}=U-\mu, \varepsilon_{-}=-\mu$ and

$$
\begin{aligned}
& \tilde{\Omega}_{\sigma}(\omega)=\Omega_{\sigma}(\omega)+\frac{V \varphi_{\sigma}}{A_{0 \sigma} A_{2,-\sigma}}, \\
& \Omega_{\sigma}(\omega)=J_{\sigma}(\omega)-\frac{R_{\sigma}(\omega)}{A_{0 \sigma} A_{2,-\sigma}} .
\end{aligned}
$$

It should be mentioned that formula (60) can be also represented in the form

$$
\left(\Xi_{\sigma}(\omega)\right)^{-1}=\left[\frac{A_{0 \sigma}}{\omega-\varepsilon_{-}-\tilde{\Omega}_{\sigma}(\omega)}+\frac{A_{2,-\sigma}}{\omega-\varepsilon_{+}-\tilde{\Omega}_{\sigma}(\omega}\right]^{-1}+\tilde{\Omega}_{\sigma}(\omega) .
$$


The relation (60) creates together with (10), (13) and (14) a set of equations for the coherent potential $J_{\sigma}(\omega)$, self-energy part $\Xi_{\sigma}(\omega)$ and Green's functions $G_{i i, \sigma}(\omega)$ and $G_{k}^{\sigma}(\omega)$.

It should be noted that the parameter $\varphi_{\sigma}$, which is expressed in terms of average values of the products of $X$ and $\xi$ operators (formula (30)), is a functional of the potential $J_{\sigma}(\omega)$. According to the spectral theorem

$$
V\left\langle X^{\sigma 0(2,-\sigma)} \xi_{\sigma}\right\rangle=\mathrm{i} \int_{-\infty}^{+\infty} \frac{\mathrm{d} \omega}{\mathrm{e}^{\beta \omega}+1}\left[V\left\langle\left\langle\xi_{\sigma} \mid X^{\sigma 0(2,-\sigma)}\right\rangle\right\rangle_{\omega+\mathrm{i} \varepsilon}-V\left\langle\left\langle\xi_{\sigma} \mid X^{\sigma 0(2,-\sigma)}\right\rangle\right\rangle_{\omega-\mathrm{i} \varepsilon}\right] .
$$

On the other hand, using the linearized equation of motion (26) and neglecting the irreducible parts, we can obtain the following system of equations

$$
\begin{aligned}
& V\left\langle\left\langle\xi_{\sigma} \mid X^{\sigma 0}\right\rangle\right\rangle\left(\omega-\varepsilon_{-}-\frac{V}{A_{0 \sigma}} \varphi_{\sigma}\right)+V^{2} \sigma \frac{\varphi_{\sigma}}{A_{2,-\sigma}}\left\langle\left\langle\xi_{\sigma} \mid X^{2,-\sigma}\right\rangle\right\rangle=\frac{A_{0 \sigma}}{2 \pi} J_{\sigma}, \\
& V^{2} \sigma \frac{\varphi_{\sigma}}{A_{0 \sigma}}\left\langle\left\langle\xi_{\sigma} \mid X^{\sigma 0}\right\rangle\right\rangle+V\left\langle\left\langle\xi_{\sigma} \mid X^{2,-\sigma}\right\rangle\right\rangle\left(\omega-\varepsilon_{+}-\frac{V}{A_{2,-\sigma}} \varphi_{\sigma}\right)=\sigma \frac{A_{2,-\sigma}}{2 \pi} J_{\sigma} .
\end{aligned}
$$

It follows herefrom

$$
\begin{aligned}
V\left\langle\left\langle\xi_{\sigma} \mid X^{\sigma 0}\right\rangle\right\rangle_{\omega} & =\frac{1}{2 \pi} \frac{J_{\sigma}(\omega)}{D_{\sigma}}\left[A_{0 \sigma}\left(\omega-\varepsilon_{+}\right)-V \frac{\varphi_{\sigma}}{A_{2,-\sigma}}\right], \\
V\left\langle\left\langle\xi_{\sigma} \mid X^{2,-\sigma}\right\rangle\right\rangle_{\omega} & =\frac{1}{2 \pi} \frac{J_{\sigma}(\omega)}{D_{\sigma}} \sigma\left[A_{2,-\sigma}\left(\omega-\varepsilon_{-}\right)-V \frac{\varphi_{\sigma}}{A_{0 \sigma}}\right] .
\end{aligned}
$$

In particular, in the $U \rightarrow \infty$ limit

$$
\begin{aligned}
V\left\langle\left\langle\xi_{\sigma} \mid X^{\sigma 0}\right\rangle\right\rangle_{\omega} & =\frac{1}{2 \pi} J_{\sigma}(\omega) \frac{A_{0 \sigma}}{\omega-\varepsilon_{-}-\frac{V \varphi_{\sigma}}{A_{0 \sigma}}}, \\
V\left\langle\left\langle\xi_{\sigma} \mid X^{2,-\sigma}\right\rangle\right\rangle_{\omega} & =0 .
\end{aligned}
$$

In this case

$$
V \varphi_{\sigma}=-V\left\langle X^{-\sigma 0} \xi_{-\sigma}\right\rangle=-\frac{1}{2 \pi} \int_{-\infty}^{+\infty} \frac{\mathrm{d} \omega}{\mathrm{e}^{\beta \omega}+1}\left[-2 \operatorname{Im} \frac{A_{0,-\sigma} J_{\sigma}(\omega)}{\omega-\varepsilon_{-}-V \frac{\varphi_{-\sigma}}{A_{0,-\sigma}}}\right]_{\omega+\mathrm{i} \varepsilon},
$$

or, in the Matsubara's representation

$$
V \varphi_{\sigma}=-\frac{1}{\beta} A_{0, \sigma} \sum_{\omega_{n}} \frac{J_{-\sigma}\left(\omega_{n}\right)}{\mathrm{i} \omega_{n}-\varepsilon_{-}-V \frac{\varphi_{-\sigma}}{A_{0, \sigma}}}
$$

Thus, we obtain a self-consistent equation for the parameter $\varphi_{\sigma}$.

Using a similar procedure one can construct an equation for $\varphi_{\sigma}$ in the case of finite values of $U$.

\section{Some specific cases}

Equations obtained in the previous section form an approximate analytical scheme of calculating both the single-site and the full electron Green's function in the framework of DMFT. Let us compare it with the standard approximations known from literature which are based on the assumption of the single-site structure of the electron self-energy. For this purpose we will consider some specific cases. 
6.1. Hubbard-I approximation $\left(J_{\sigma}=0, R_{\sigma}=0, \varphi_{\sigma}=0 ; \tilde{\Omega}_{\sigma}=0\right.$ )

It is the simplest approximation; renormalization of energies of atomic electron transitions is absent and the scattering processes via coherent potential are not taken into account. The expression for the single-site self-energy part

$$
\Xi_{\sigma}(\omega)=\frac{A_{0 \sigma}}{\omega-\varepsilon_{-}}+\frac{A_{2,-\sigma}}{\omega-\varepsilon_{+}}
$$

corresponds to the Hubbard-I approximation [22]. Electron energy spectrum described by Green's function $G_{k}^{\sigma}(\omega)$ consists in this case of two Hubbard subbands divided by a gap existing at any relationship between the values of $U$ and $t$ parameters.

\subsection{Static mean-field approximation $\left(J_{\sigma}=0, \quad R_{\sigma}=0 ; \quad \tilde{\Omega}_{\sigma}=B \equiv\right.$ $\left.V \varphi_{\sigma} / A_{0 \sigma} A_{2,-\sigma}\right)$}

In this case only a self-consistent shift of the energy levels of the single-site atomic problem is taken into account. The coherent potential $J_{\sigma}(\omega)$ is replaced in the expression for $\varphi_{\sigma}$ by the approximate expression

$$
J_{\sigma}(\omega)=\sum_{k j} t_{i k} t_{i j} G_{k j, \sigma}(\omega)
$$

following from (15) when the difference between $G_{k j, \sigma(i)}$ and $G_{k j, \sigma}$ is neglected.

The expression for the self-energy part

$$
\left[\Xi_{\sigma}(\omega)\right]^{-1}=\left[G_{\sigma}^{a}(\omega)\right]^{-1}=\left[\frac{A_{0 \sigma}}{\omega-\varepsilon_{-}-B}+\frac{A_{2,-\sigma}}{\omega-\varepsilon_{+}-B}\right]^{-1}+B,
$$

that can be obtained in this case, corresponds to the summation of the series

$$
\hat{G}=\hat{g}_{0} \hat{I}-\hat{g}_{0} \hat{W} \hat{g}_{0} \hat{I}+\hat{g}_{0} \hat{W} \hat{g}_{0} \hat{W} \hat{g}_{0} \hat{I}-\ldots,
$$

where

$$
\begin{gathered}
\hat{g}_{0}=\left(\begin{array}{cc}
\left(\omega-\varepsilon_{-}\right)^{-1} & 0 \\
0 & \left(\omega-\varepsilon_{+}\right)^{-1}
\end{array}\right), \quad \hat{I}=\frac{1}{2 \pi}\left(\begin{array}{cc}
A_{0 \sigma} & 0 \\
0 & A_{2,-\sigma}
\end{array}\right), \\
\hat{W}=B\left(\begin{array}{cc}
-A_{2,-\sigma} & A_{0 \sigma} \\
A_{2,-\sigma} & -A_{0 \sigma}
\end{array}\right) .
\end{gathered}
$$

A sum of the diagrams with loop-like inclusions into the line of the electron singlesite Green's function corresponds to this series in a diagrammatic representation. Such inclusions lead to the renormalization of energies of the electron levels [23]. In particular, at $U=\infty$

$$
G_{\sigma}^{(a)}(\omega)=\left\langle\left\langle X^{0 \sigma} \mid X^{\sigma 0}\right\rangle\right\rangle_{\omega}=\frac{1}{2 \pi} \frac{A_{0 \sigma}}{\omega-\varepsilon_{-}-V \frac{\varphi_{-\sigma}}{A_{0 \sigma}}} .
$$


Energy shift of the electron transition

$$
\Delta \varepsilon_{-}=A_{2,-\sigma} B=-\frac{1}{A_{0 \sigma}} \frac{1}{\beta} \sum_{\omega_{n}} \sum_{k} t_{i k}\left\langle X_{k}^{-\sigma, 0} X_{i}^{0,-\sigma}\right\rangle
$$

coincides in this case with the previously obtained one in a number of papers (see, for example $[8,24,25]$ ) using a more complicated (in comparison with Hubbard-I approximation) decoupling procedure in equations for the Green's function $G_{k}^{\sigma}(\omega)$.

\subsection{Hubbard-III approximation ( $\left.\varphi_{\sigma}=0 ; \tilde{\Omega}_{\sigma}=J_{\sigma}-R_{\sigma} / A_{0 \sigma} A_{2,-\sigma}\right)$}

We can pass on to this approximation neglecting, at first, the renormalization of the atomic electron levels and approximating, secondly, in the case of the half filling (when $\left\langle X^{00}\right\rangle=\left\langle X^{22}\right\rangle$ ) in the expression $\left(G_{1}\right)$

$$
\frac{\left\langle X^{\sigma \sigma}\right\rangle}{A_{0 \sigma} A_{2,-\sigma}} \rightarrow 1, \quad \frac{\left\langle X^{22}\right\rangle}{A_{0 \sigma} A_{2,-\sigma}} \rightarrow 1
$$

which becomes exact only in the $U \rightarrow 0$ limit. Consequently, an effective potential of dynamical mean field $\tilde{\Omega}_{\sigma}(\omega)$ takes the form

$$
\tilde{\Omega}_{\sigma}(\omega)=J_{\sigma}(\omega)+J_{-\sigma}(\omega)-J_{-\sigma}(U-2 \mu-\omega) .
$$

It corresponds (together with the expression (60) for the electron self-energy) to the Hubbard-III approximation [10]. A potential $\tilde{\Omega}_{\sigma}(\omega)$ includes (besides the coherent potential $\left.J_{\sigma}(\omega)\right)$ the terms which describe a scattering on the spin and charge fluctuations. Electron energy spectrum consists in this case of two subbands only at $U>U_{\text {c }}$ where the critical value $U_{\mathrm{c}}$ corresponds to the metal-insulator transition.

\subsection{Alloy-analogy (AA) approximation $\left(R_{\sigma}=0, \varphi_{\sigma}=0 ; \tilde{\Omega}_{\sigma}(\omega)=J_{\sigma}(\omega)\right)$}

The scattering processes are taken here into account only via coherent potential. The single-site Green's function looks like

$$
G_{i i, \sigma}(\omega)=G_{\sigma}^{(a)}(\omega)=\frac{A_{0 \sigma}}{\omega-\varepsilon_{-}-J_{\sigma}}+\frac{A_{2,-\sigma}}{\omega-\varepsilon_{+}-J_{\sigma}}
$$

in this case. This expression is analogous to the locator function for a binary alloy [16]. The procedure of the $G_{k}^{\sigma}(\omega)$ function calculation corresponds to the CPA method. Let us write for this approximation an irreducible, according to Dyson, self-energy part $\Sigma_{\sigma}=\omega-\varepsilon_{-}-\left(\Xi_{\sigma}\right)^{-1}$, using the expression (60) at $\tilde{\Omega}_{\sigma}=J_{\sigma}$ :

$$
\Sigma_{\sigma}=A_{2,-\sigma} U /\left(1-\frac{A_{0 \sigma} U}{\omega+\mu-J_{\sigma}}\right) .
$$

Or, after excluding a coherent potential

$$
\Sigma_{\sigma}(\omega)=\frac{A_{2,-\sigma} U}{1-G_{i i}^{\sigma}(\omega)\left(U-\Sigma_{\sigma}(\omega)\right)} .
$$

This equation corresponds to the alloy-analogy (AA) approximation [8]. 


\subsection{Modified alloy-analogy (MAA) approximation ( $R_{\sigma}=0$; $\left.\tilde{\Omega}_{\sigma}=J_{\sigma}+V \varphi_{\sigma} / A_{0 \sigma} A_{2, \sigma} \equiv J_{\sigma}+B\right)$}

An AA-approach is supplemented here by the inclusion of renormalization of single-site electron levels. It can now be obtained that

$$
\Sigma_{\sigma}=A_{2,-\sigma} U /\left(1-\frac{A_{0 \sigma} U}{\omega-\varepsilon_{-}-\tilde{\Omega}}\right) .
$$

This relation can be transformed into the equation

$$
\Sigma_{\sigma}(\omega)=A_{2,-\sigma} U /\left(1-\frac{G_{i i, \sigma}\left(U-\Sigma_{\sigma}\right)}{1-G_{i i, \sigma} B}\right)
$$

known in the so-called Modified AA-approach [8,11].

One can see from the quoted specific cases that the approach developed in this work includes a number of known approximations giving in addition their unification and generalization. The proposed scheme is more complete than Hubbard-III approximation (which in its turn is the most general of the quoted ones) and differs from it by the allowance for a self-consistent renormalization (due to the static internal field) of the local energy spectrum as well as by the modification of the potential $\tilde{\Omega}_{\sigma}$ constituent parts to a more elaborated inclusion of the magnon and charge fluctuation scattering processes. Participation of Bose-particles in such a scattering is taken into account in our scheme in a more consistent way.

Quantitative changes in the electron spectrum (in particular, in the electron density of states) and then in the thermodynamics of the model, that might be the consequence of applying the approach suggested herein, can be the subject of subsequent calculations with the use of numerical methods.

\section{Simple applications of the method}

Let us demonstrate here the potentialities of the developed approximative scheme using the examples of two models (the Falicov-Kimball model and the simplified pseudospin-electron model) which are analytically solvable in the DMFT approach.

\subsection{Falicov-Kimball model}

It is supposed in this model [17] (which can be considered as a specific case of the Hubbard model) that the electron transfer from one lattice site to another one takes place only in the case of the only $(\sigma=+)$ orientation of spins. Electrons with the opposite spin orientation $(\sigma=-)$ remain localized. They can effect the energy of delocalized electrons being a source of scattering.

The Hamiltonian of the effective single-site problem looks in this model like

$$
\begin{aligned}
\tilde{H}_{\mathrm{eff}}= & (U-2 \mu) X^{22}-\mu \sum_{\sigma} X^{\sigma \sigma} \\
& +V\left[\xi_{\uparrow}^{+}\left(X^{0,+}+X^{-, 2}\right)+\left(X^{+0}+X^{2-}\right) \xi_{\uparrow}\right]+H_{\xi} .
\end{aligned}
$$


In this case, in the equations of motion for $X$-operators

$$
\begin{aligned}
{\left[X^{0+}, \tilde{H}_{\mathrm{eff}}\right] } & =-\mu X^{0+}+V\left(X^{00}+X^{++}\right) \xi_{\uparrow}, \\
{\left[X^{-, 2}, \tilde{H}_{\mathrm{eff}}\right] } & =(U-\mu) X^{-, 2}+V\left(X^{22}+X^{--}\right) \xi_{\uparrow} .
\end{aligned}
$$

those terms are absent which are responsible for the scattering with the participation of Bose-particles (magnons and charge excitations). Only the components

$$
Z^{0+}=V \overparen{\left(X^{00}+X^{++}\right) \xi_{\uparrow}}, \quad Z^{-, 2}=V \overparen{\left(X^{22}+X^{--}\right) \xi_{\uparrow}}
$$

of irregular parts of the time derivatives of $X$-operators are present.

The corresponding irreducible Green's functions are equal to

$$
\begin{aligned}
2 \pi\left\langle\left\langle Z^{0+} \mid Z^{+0}\right\rangle\right\rangle_{\omega} & =V^{2} A_{0+} \cdot 2 \pi G_{\uparrow}(\omega)=A_{0+} J_{\uparrow}(\omega), \\
2 \pi\left\langle\left\langle Z^{-, 2} \mid Z^{2-}\right\rangle\right\rangle_{\omega} & =V^{2} A_{-, 2} \cdot 2 \pi G_{\uparrow}(\omega)+A_{-, 2} J_{\uparrow}(\omega), \\
\left\langle\left\langle Z^{0+} \mid Z^{2-}\right\rangle\right\rangle_{\omega} & =\left\langle\left\langle Z^{-, 2} \mid X^{+0}\right\rangle\right\rangle_{\omega}=0 .
\end{aligned}
$$

Using now the formulae (38)-(40) we obtain

$$
G_{\uparrow}^{(a)}(\omega)=\frac{A_{0+}}{\omega+\mu-J_{\uparrow}(\omega)}+\frac{A_{-, 2}}{\omega+\mu-U-J_{\uparrow}(\omega)} .
$$

This expression has the same structure as the Green's function for the AA-approximation and is exact for the Falicov-Kimball model (see, for example, [14]).

\subsection{Simplified pseudospin-electron model}

In recent years the pseudospin-electron model (PEM) has been among the actively investigated models in the theory of strongly correlated electron systems. In addition to the Hubbard type correlation, an interaction with locally anharmonic lattice vibrations is included into the model. The corresponding degrees of freedom are described by the pseudospin variables with $S=1 / 2$. The Hamiltonian of the PEM has the form [26] analogous to (1) with

$$
H_{i}=U n_{i \uparrow} n_{i \downarrow}-\mu \sum_{\sigma} n_{i \sigma}+g \sum_{\sigma} n_{i \sigma} S_{i}^{z}-h S_{i}^{z}+\Omega S_{i}^{x}
$$

(here $h$ is internal asymmetry field; $\Omega$ is a parameter of the tunnelling type splitting).

One can put $U=0$ and $\Omega=0$ for simplification. Such a situation was considered in $[18,27]$. It is possible to analytically solve an effective single-site problem for this case too.

The effective Hamitlonian for the simplified model is as follows

$$
\tilde{H}_{\text {eff }}=-\mu \sum_{\sigma} n_{\sigma}+g \sum_{\sigma} n_{\sigma} S^{z}-h S^{z}+V \sum_{\sigma}\left(\xi_{\sigma}^{+} a_{\sigma}+a_{\sigma}^{+} \xi_{\sigma}\right)+H_{\xi} .
$$

Let us write the required single-site Green's function in the form

$$
G_{\sigma}^{(a)}(\omega)=\left\langle\left\langle P^{+} a_{\sigma} \mid P^{+} a_{\sigma}^{+}\right\rangle\right\rangle_{\omega}+\left\langle\left\langle P^{-} a_{\sigma} \mid P^{-} a_{\sigma}^{+}\right\rangle\right\rangle_{\omega},
$$


where $P^{ \pm}=1 / 2 \pm S^{z}$ are the operators projecting into states with a given pseudospin orientation.

Following the procedure described in section 3 we consider the equation of motion.

$$
\left[P^{ \pm} a_{\sigma}, \tilde{H}_{\mathrm{eff}}\right]=E^{ \pm} P^{ \pm} a_{\sigma}+V P^{ \pm} \xi_{\sigma}
$$

where $E^{ \pm}=E_{0} \pm g / 2$. The irregular part in this case is

$$
Z^{ \pm}=V \overparen{P^{ \pm} \xi_{\sigma}} \equiv V P^{ \pm} \xi_{\sigma}
$$

The different-time decoupling gives

$$
\left\langle\langle\overbrace{P^{ \pm} \xi_{\sigma}} \mid \overbrace{P^{ \pm} \xi_{\sigma}^{+}}\rangle\right\rangle_{\omega}=\left\langle P^{ \pm}\right\rangle\left\langle\left\langle\xi_{\sigma} \mid \xi_{\sigma}^{+}\right\rangle\right\rangle_{\omega}
$$

As a result, we obtain Dyson equation

$$
G_{\sigma}^{ \pm}(\omega)=G_{0}^{ \pm}+G_{0}^{ \pm} M_{\sigma}^{ \pm} G_{\sigma}^{ \pm}
$$

with

$$
G_{0}^{ \pm}=\frac{\left\langle P^{ \pm}\right\rangle}{\omega-E^{ \pm}}, \quad M_{\sigma(\omega)}^{ \pm}=\frac{1}{\left\langle P^{ \pm}\right\rangle} J_{\sigma}(\omega)
$$

It follows herefrom that

$$
G_{\sigma}^{(a)}(\omega)=\frac{\left\langle P^{+}\right\rangle}{\omega-E^{+}-J_{\sigma}(\omega)}+\frac{\left\langle P^{-}\right\rangle}{\omega-E^{-}-J_{\sigma}(\omega)} .
$$

This expression coincides with the one obtained in the $d=\infty$ limit in the framework of DMFT [18]. The different-time decoupling (93) is an exact procedure in this case.

Let us mention that the two-pole structure of the single-site Green's function leads to the effect of the metal-insulator transition type at $t \sim g$. In the case $t>g$ the electron spectrum consists of one broad band while at $t<g$ there appears a gap and the splitting into two Hubbard bands takes place [18].

\section{Concluding remarks}

The developed approach to the analytic solution of the effective single-site problem in the DMFT method is an approximate interpolating scheme that in specific cases includes a number of known approximations for the Hubbard model and similar models. The scheme is based on the application of the equations of motion method and the technique of the irreducible Green's functions with the projection onto the single-site Hubbard basis of the Fermi-type operators. The examples are given where the proposed approach gives exact results.

The simplicity and availability of this scheme make it attractive for the approximate analytical consideration. It seems useful to apply it to the problems which have been considered up to now by means of numerical methods. At the same time an accuracy that can be achieved in the framework of the proposed scheme can 
be established after comparing the results of calculations of the parameters of the electron spectrum and thermodynamical functions.

Let us mention that the question of calculating the electron mean occupation numbers (and deriving an equation for chemical potential) as well as determining the grand canonical potential was not elucidated in this work. It will be the subject of a separate publication.

\section{A. Statistical operator for the effective single-site problem}

Thermodynamic perturbation theory for the effective single-site problem with the Hamiltonian $\tilde{H}_{\text {eff }}$ can be formulated based on the interaction representation for the statistical operator with the use of the $H_{0}+H_{\xi}$ operator as the zero-order Hamiltonian:

$$
\mathrm{e}^{-\beta \tilde{H}_{\mathrm{eff}}}=\mathrm{e}^{-\beta\left(H_{0}+H_{\xi}\right)} \sigma(\beta) \equiv \mathrm{e}^{-\beta\left(H_{0}+H_{\xi}\right)} \mathrm{T}_{\tau} \exp \left\{-\int_{0}^{\beta} \mathrm{d} \tau \sum_{\sigma} V \hat{Q}_{\sigma}(\tau)\right\},
$$

where

$$
\hat{Q}_{\sigma}(\tau)=a_{\sigma}^{+}(\tau) \xi_{\sigma}(\tau)+\xi_{\sigma}^{+}(\tau) a_{\sigma}(\tau) .
$$

Let us average over the variables of the auxiliary Fermi-field

$$
\langle\sigma(\beta)\rangle_{\xi}=\frac{1}{Z_{\xi}} \operatorname{Sp~e}^{-\beta H_{\xi}} \sigma(\beta),
$$

using the semi-invariant expansion. As a result we will come to the statistical operator for a given atom

$$
\hat{\rho}^{(a)}=\frac{1}{Z^{(a)}} \mathrm{e}^{-\beta H_{0}} \mathrm{~T}_{\tau} \exp \left\{\sum_{n=2}^{\infty} \frac{V^{n}}{n !} M_{n}\left(a^{+}, a\right)\right\} .
$$

Here only the even order semi-invariants are presented

$$
\begin{aligned}
M_{2}\left(a^{+}, a\right) & =\int_{0}^{\beta} \mathrm{d} \tau_{1} \int_{0}^{\beta} \mathrm{d} \tau_{2} \sum_{\sigma_{1} \sigma_{2}}\left\langle T_{\tau} \hat{Q}_{\sigma_{1}}\left(\tau_{1}\right) \hat{Q}_{\sigma_{2}}\left(\tau_{2}\right)\right\rangle_{\xi}^{\mathrm{c}}, \\
M_{4}\left(a^{+}, a\right) & =\int_{0}^{\beta} \mathrm{d} \tau_{1} \ldots \int_{0}^{\beta} \mathrm{d} \tau_{4} \sum_{\sigma_{1} \ldots \sigma_{4}}\left\langle T_{\tau} \hat{Q}_{\sigma_{1}}\left(\tau_{1}\right) \ldots \hat{Q}_{\sigma_{4}}\left(\tau_{4}\right)\right\rangle_{\xi}^{\mathrm{c}},
\end{aligned}
$$

etc.; after the averaging over the $\xi$-subsystem they are the operators acting on the atomic electron states.

Introducing the Green's function

$$
-\left\langle T_{\tau} \xi_{\sigma_{1}}\left(\tau_{1}\right) \xi_{\sigma_{2}}^{+}\left(\tau_{2}\right\rangle_{\xi}=\delta_{\sigma_{1} \sigma_{2}} \mathcal{G}_{\sigma_{1}}\left(\tau_{1}-\tau_{2}\right)\right.
$$

and applying the Wick's theorem we can see that only the semi-invariant of the second order exists

$$
M_{2}\left(a^{+}, a\right)=-\sum_{\sigma} \int_{0}^{\beta} \mathrm{d} \tau_{1} \int_{0}^{\beta} \mathrm{d} \tau_{2} \mathcal{G}_{\sigma}\left(\tau_{1}-\tau_{2}\right) a_{\sigma}^{+}\left(\tau_{1}\right) a_{\sigma}\left(\tau_{2}\right),
$$


while all semi-invariants of the higher order are equal to zero

$$
M_{4}\left(a^{+}, a\right)=M_{6}\left(a^{+}, a\right)=\ldots=0 .
$$

(the $\xi$-subsystem with the Hamiltonian $H_{\xi}$ is defined as an ideal one).

Finally we obtain

$$
\hat{\rho}^{(a)}=\frac{1}{Z^{(a)}} \mathrm{e}^{-\beta H_{0}} \mathrm{~T}_{\tau} \exp \left\{-\sum_{\sigma} \int_{0}^{\beta} \mathrm{d} \tau_{1} \int_{0}^{\beta} \mathrm{d} \tau_{2} J_{\sigma}\left(\tau_{1}-\tau_{2}\right) a_{\sigma}^{+}\left(\tau_{1}\right) a_{\sigma}\left(\tau_{2}\right)\right\},
$$

where the notation $V^{2} \mathcal{G}_{\sigma}\left(\tau_{1}-\tau_{2}\right)=J_{\sigma}\left(\tau_{1}-\tau_{2}\right)$ is introduced.

\section{References}

1. Metzner W. // Phys. Rev. B, 1991, vol. 43, p. 8549.

2. Metzner W., Vollhardt D. // Phys. Rev. Lett., 1989, vol. 62, p. 324.

3. Müller-Hartmann E. // Z. Phys. B, 1989, vol. 74, p. 507.

4. Georges A., Kotliar G. // Phys. Rev. B, 1992, vol. 45, p. 6479.

5. Jarrel M. // Phys. Rev. Lett., 1992, vol. 69, p. 168.

6. Janis V., Vollhardt D. // Int. J. Mod. Phys., B, 1992, vol. 6, p. 731.

7. Georges A., Kotliar G., Krauth W., Rosenberg M.J. // Rev. Mod. Phys., 1996, vol. 68, p. 13.

8. Potthoff M., Herrmann T., Wegner T., Nolting W. // Phys. Stat. Sol. (b), 1998, vol. 210, p. 199.

9. Gebhard F. The Mott Metal-Insulator transition. Springer Tracts in Modern Physics, vol. 137, Berlin, Springer, 1997.

10. Hubbard J. // Proc. Roy. Soc. A, 1964, vol. 281, p. 401.

11. Herrmann T., Nolting W. // Phys. Rev. B, 1996, vol. 53, p. 10579.

12. van Dongen P.G.J. // Phys. Rev. B, 1994, vol. 50, p. 14016.

13. Hettler M.H., Tahvildar-Zadeh A.N., Jarrel M. // Phys. Rev. B, 1998, vol. 58, p. 7475.

14. Brandt U., Mielsch C. // Z. Phys. B, 1989, vol. 75, p. 365; 1990, vol. 79, p. 295; 1991, vol. 82 , p. 37.

15. Velicki B., Kirpatrick S., Ehrenreich H. // Phys. Rev., 1968, vol. 175, p. 741.

16. Ehrenreich H., Schwartz L.M. // Solid State Physics, 1976, vol. 31, p. 150.

17. Falicov L.M., Kimball J.C. // Phys. Rev. Lett., 1969, vol. 22, p. 997.

18. Stasyuk I.V., Shvaika A.M. // J. Phys. Studies, 1999, vol. 3, p. 177.

19. Tserkovnikov Yu.A. // Teor. i Mat. Fiz., 1971, vol. 7, p. 260.

20. Plakida N.M. // Phys. Lett. A, 1973, vol. 43, p. 471.

21. Plakida N.M. Method of two-time Green's functions in the theory of anharmonic crystals. - In: Statistical physics and quantum field theory, ed. by N.N. Bogolyubov, Moscow, 1973, p. 205-240.

22. Hubbard J. // Proc. Roy. Soc. A, 1963, vol. 276, p. 238.

23. Stasyuk I.V., Danyliv O.D. // Phys. Stat. Sol. (b) (in print).

24. Harris A.B., Lange R.V. // Phys. Rev., 1967, vol. 157, p. 295.

25. Plakida N.M., Yushankov V.Yu., Stasyuk I.V. // Physica C, 1989, vol. 160, p. 80.

26. Müller K.A. // Z. Phys. B, 1990, vol. 80, p. 193.

27. Stasyuk I.V., Shvaika A.M., Tabunshchyk K. // Cond. Matt. Phys., 1999, vol. 2, p. 109. 


\title{
Наближена аналітична схема методу динамічного середнього поля для сильно скорельованих електронних систем
}

\author{
І.В.Стасюк \\ Інститут фізики конденсованих систем НАН України, \\ 79011 Львів, вул. Свєнціцького, 1 \\ Отримано 17 березня 2000 р.
}

У роботі розроблена наближена аналітична схема методу динамічного середнього поля (МДСП), який використовується для електронних систем з хаббардівськими кореляціями і $€$ точним при безмежній розмірності простору. Ефективна одновузлова задача, що виникає в цьому методі, сформульована за допомогою допоміжного ферміполя. Для її розв'язання використано техніку незвідних функцій Гріна при проектуванні на хаббардівський базис фермі-операторів. Отримано систему рівняннь МДСП у наближенні, яке $є$ узагальненням наближення Хаббарда-III і поєднує його із самоузгодженим перенормуванням локальних електронних рівнів. Показано, що ряд відомих наближень, що базуються на одновузловій структурі власноенергетичної частини електронної функції Гріна, є простими частинними випадками запропонованої схеми.

Ключові слова: сильні електронні кореляції, модель Хаббарда, теорія динамічного середнього поля, допоміжне фермі-поле, незвідні функції Гріна

PACS: $71.10 . \mathrm{Fd}, 05.30 . \mathrm{FK}$ 